\title{
On the Feynman Ratchet and the Brownian Motor
}

\author{
Gyula Vincze'1, Gyula Peter Szigeti², Andras Szasz ${ }^{1}$ \\ ${ }^{1}$ Department of Biotechnics, St. Istvan University, Budapest, Hungary \\ ${ }^{2}$ Institute of Human Physiology and Clinical Experimental Research, Semmelweis University, Budapest, Hungary \\ Email: biotech@gek.szie.hu
}

How to cite this paper: Vincze, Gy., Szigeti, Gy.P. and Szasz, A. (2018) On the Feynman Ratchet and the Brownian Motor. Open Journal of Biophysics, 8, 22-30.

https://doi.org/10.4236/ojbiphy.2018.81003

Received: October 18, 2017

Accepted: January 7, 2018

Published: January 10, 2018

Copyright $\odot 2018$ by authors and Scientific Research Publishing Inc. This work is licensed under the Creative Commons Attribution International License (CC BY 4.0).

http://creativecommons.org/licenses/by/4.0/

\begin{abstract}
We study the Brownian ratchet conditions starting with Feynman's proposal. We show that this proposal is incomplete, and is in fact non-workable. We give the correct model for this ratchet.
\end{abstract}

\section{Keywords}

Brownian Motor, Feynman Ratchet, Self-Sustaining Oscillation

\section{Introduction}

The theory of material transport driven by fluctuations was worked out to explain motor proteins [1]. These molecular motors constrain the motion dominantly in one-dimensional surmount energy wells and barriers. While the obstacles limit diffusion, thermal noise is a part of the thermal activation [2]. The directed motion is energized by the fluctuations of the height of the barrier, depending on the external modulation, and is coupled to the ATP-hydrolysis as a chemical energy source. The external non-equilibrium fluctuation could make macroscopic displacement in a random movement of a particle in a space-periodic T-temperature sample. The periodic potential is the model of the biopolymer that drives the movement in one dimension. The nonequilibrium fluctuation, which is usually quasi-periodic, is massively energized by the ATP-ADP reaction. The inspiration for this theoretical model of the whole phenomenon was the Feynman's ratchet [3].

\section{Method}

The Feynman ratchet [3] is a heat-engine, having two heat-reservoirs with different temperatures $\left(T_{1}\right.$ and $\left.T_{2} ; T_{2}>T_{1}\right)$. The reservoirs are connected with a 
well fit axis, which is supposed to be frictionless. The axis has a blade-wheel in one end, immersed into the higher temperature, while a ratchet on the other (lower temperature) heat-reservoir [4] [5], (see Figure 1). According to Feynman, the engine rotates due to the different equilibrium fluctuations of the two heat-reservoirs. This ratchet heat-engine demonstrates of the efficiency equal to the hypothetical Carnot engine.

Unfortunately, this construction does not work, and could not demonstrate the Carnot-engine. The problem is that when the blade-wheel is in thermal equilibrium with the heat-reservoir, the internal energy fluctuates between them, but its time-average is zero. The blade-wheel cannot provide work, because contradicts to the first law of thermodynamics. Feynman's mistake was to ignore the inherent behavior of the Carnot cycle: the Carnot cycle is a non-equilibrium process in a thermodynamic sense. While the Carnot engine works, the internal energy flows from the heat reservoir of higher temperature to lower one. In Feynman ratchet no such heat-flow is supposed.

Feynman's proposal could be realistic when the internal energy can flow only through the axis of the engine between the two reservoirs, so both the blade and the ratchet are places of non-equilibrium fluctuations. The blade absorbs energy, while the ratchet wheel provides it to the heat reservoir with lower temperature. With this "extra" condition, the process became non-equilibrium and the device (in principle) can work. The experimental "realization" shows the non-equilibrium effect and the wheel rotates [7].

To work out the mathematical model, let us simplify the machine as a threepole thermal construction. This exchanges energy and entropy in these three poles (Figure 2).

Supposing the quasi-stationer operation the first law of thermodynamics requests:

$$
I_{u 1}-I_{u 2}=P
$$

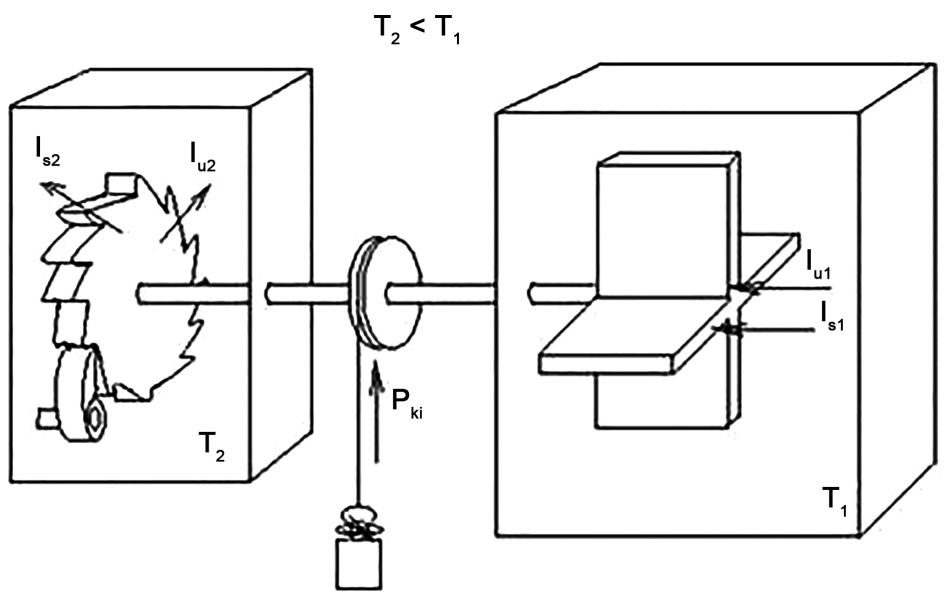

Figure 1. The Feynman heat-engine. (It is an interesting fact of science history that the publisher first refused to publish this part, but Feynman was insisting it be published) (Figure is a modified sketch form [6]). 


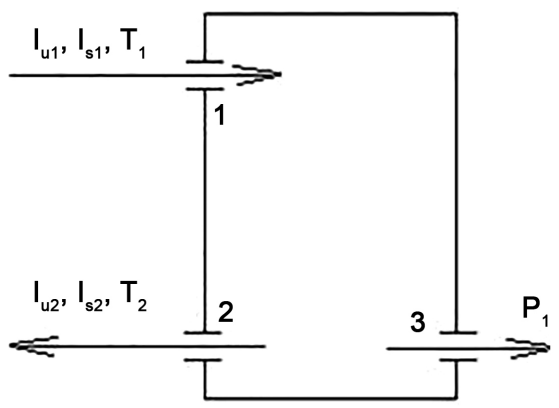

Figure 2. Feynman's heat engine as a threepole. (Two of them are connected to the reservoir exchanges, while the third is the environmental effect, showing the output power $\mathrm{P})$.

The currents of entropy from the second law of thermodynamics are

$$
I_{s 1}=\frac{I_{u 1}}{T_{1}}, \quad I_{s 2}=\frac{I_{u 2}}{T_{2}}
$$

The energy-dissipation, also according to the second law, is:;

$$
I_{s 1}-I_{s 2}=\Sigma_{s}>0
$$

And so the efficiency of the engine is:

$$
\eta:=\frac{P_{k i}}{I_{u 1}}=1-\frac{T_{2}}{T_{1}}\left(1-\frac{\Sigma_{s}}{I_{s 1}}\right)
$$

Introducing the effective temperature:

$$
T_{1}^{*}=\frac{T_{1}}{\left(1-\frac{\Sigma_{s}}{I_{s 1}}\right)}
$$

We have an equivalent mathematical form with the Carnot efficiency:

$$
\eta:=\frac{P_{k i}}{I_{u 1}}=1-\frac{T_{2}}{T_{1}^{*}}
$$

The entropy production from the equations above is:

$$
\Sigma_{s}=-I_{u 1}\left(\frac{1}{T_{1}}-\frac{1}{T_{1}^{*}}\right)>0
$$

Based on Onsager's law, we can write the equation of the heat exchange between the blade and the reservoir using the Fourier heat conduction law, where $T_{1}^{*}$ is the definite temperature at the blade surface:

$$
I_{u 1}=-k\left(T_{1}^{*}-T_{1}\right)
$$

Hence the output power $P$ could be constructed by combination of Equations (5)-(8):

$$
P=k \eta\left(T_{1}-\frac{T_{2}}{1-\eta}\right)
$$


It is clear from Equation (9), when the efficacy is the same as the Carnot cycle, then no output power could be detected. In this case $I_{u 1}=0$ also. This was shown in the literature, i.e. zero heat flow at maximum efficiency [8]. To calculate the efficiency belonging to the maximum output power we see that the maximum power is:

$$
P_{\max }=k\left(\sqrt{T_{1}}-\sqrt{T_{2}}\right)^{2}
$$

And in this case the (optimal) efficiency is:

$$
\eta_{\text {opt }}=1-\sqrt{\frac{T_{2}}{T_{1}}}
$$

Its connection to the Carnot efficient is:

$$
\eta_{\text {opt }}=\frac{\eta_{\text {Car }}}{\left(1+\sqrt{\frac{T_{2}}{T_{1}}}\right)}
$$

Consequently,

$$
\frac{\eta_{\text {Car }}}{2}<\eta_{\text {opt }}<\eta_{\text {Car }}
$$

Note that these two equations (Equations (12) and (13)) have been obtained for dissipative heat-engines by heuristic considerations [9] too. The conclusion about Feynman's heat engine is that it is pumped by the non-equilibrium fluctuations and its efficiency cannot be the same as that for the Carnot cycle, except when it has no output power. The Feynman engine with output power has smaller efficacy than the ideal Carnot one.

However, these purely thermodynamic considerations give no idea how the machine could work, only we have shown, that it does not contradict the first law of thermodynamics. The question remains how could the Feynman ratchet engine work?

One explanation could be that the internal energy flow on the axis is the phonon-dominant flow. Phonons have momentum, generating a momentum flow from the higher temperature reservoir to the lower one. When the tongue of the ratchet is touching only on the edge of the ratchet-wheel, then the phonon flow leaves the wheel by radial symmetry, and the flow is isotropic. In this case, the resulting angular momentum will be zero. When the tongue touches the wheel, it cannot move (i.e. rectification of the rotation direction). However, when the tongue of the ratchet has non-equilibrium fluctuations, then moves away from the wheel, and it cools down its previous place, because energy has been removed from its fluctuations. In this case a greater number of phonons will leave the wheel, because the heat flow also will be higher to this spot. In this situation, the wheel has a non-equilibrium thermal situation, and the larger phonon-output will give angular momentum and thus turn the axis. This "rocket-style" propulsion will change the momentum in the place of the inhomogeneity. A better engine solution could be a Segner-wheel, which will be thermally isolated 
on its surface: only the tip of the arms of the wheel would be a good thermal conductor. Note however that there is no mechanical rectifier.

\section{Result}

We first study a saw-tooth-shaped potential infinite shape in space and "breathing" in time between a minimum and maximum. This would be the analogue of the ratchet-blade-wheel composition constructed by Feynman. This "flashing ratchet" [10] does not work when the signal is deterministic and periodic, but is also inoperable when the signal is stochastic but mean-square (ms) differentiable. This is not the case for [10] and others where the time-fluctuation of the potential is random. However, we show that these models are too complicated to evaluate mathematically, and the possible constructions of the various fluctuations could be contentious.

Let us first study the deterministic, periodic modulation signals. The movements of a particle could be described by the following Langevin equation:

$$
\gamma \dot{x}=-\partial_{x} V(x, t)+\gamma \sqrt{2 D} \xi(t)
$$

where the $V(x, t)$ is the potential, periodic in space and time, and $\xi(t)$ is the stochastic force from different sources like heat. Due to the large damping, this approach is accepted in biophysical research. Connected to this we write that:

$$
\begin{aligned}
& \partial_{t} P(x, t)+\partial_{x} j(x, t)=0, \\
& j(x, t)=-\frac{k T}{\gamma} \mathrm{e}^{-\frac{V(x, t)}{k T}} \partial_{x} \mathrm{e}^{\frac{V(x, t)}{k T}} P(x, t)
\end{aligned}
$$

i.e. the Fokker-Planck-equations.

$P(x, t)$ is periodic in both its variables in the case of stationary process. Consequently considering the time-average of the continuity Equation (15), we obtain:

$$
\left\langle\partial_{t} P(x, t)\right\rangle_{t}+\left\langle\partial_{x} j(x, t)\right\rangle=\left\langle\partial_{t} P(x, t)\right\rangle_{t}+\partial_{x}\langle j(x, t)\rangle=0 \rightarrow \partial_{x}\langle j(x, t)\rangle=0
$$

From here the time-average of the current density of the probability does not depend on space. Hence the current density could depend only on time, so the constitutive equation is:

$$
j(t)=-\frac{k T}{\gamma} \mathrm{e}^{-\frac{V(x, t)}{k T}} \partial_{x} \mathrm{e}^{\frac{V(x, t)}{k T}} P(x, t)
$$

By rearranging and integration on the space period:

$$
j(t) \int_{x}^{x+\lambda} \frac{\gamma}{k T} \mathrm{e}^{\frac{V(x, t)}{k T}} \mathrm{~d} x=\int_{x}^{x+\lambda} \partial_{x} \mathrm{e}^{\frac{V(x, t)}{k T}} P(x, t) \mathrm{d} x=\left[\mathrm{e}^{\frac{V(x, t)}{k T}} P(x, t)\right]_{x}^{x+\lambda}
$$

Equation (18) means that no particle transport could happen at these conditions, because the right-hand side is due to the space periodicity zero, there is a positive function in the integral at the left side of the equation, and consequently only the current density of probability could be zero. The fine structure of the 
transport could be obtained by rearranging Equation (15):

$$
j(x, t)=\frac{F(x, t)}{\gamma} P(x, t)-\frac{k T}{\gamma} \partial_{x} P(x, t)
$$

where $F(x, t)=\partial_{x} V(x, t)$ is the force The space average will be:

$$
\langle j(x, t)\rangle_{x}=\left\langle\frac{F(x, t)}{\gamma} P(x, t)\right\rangle_{x}-\frac{k T}{\gamma}\left\langle\partial_{x} P(x, t)\right\rangle_{x}
$$

Due to the zero value of the left side and zero of the second term in the right, the average of the convective current density in space is zero as well.

$$
\left\langle\frac{F(x, t)}{\gamma} P(x, t)\right\rangle_{x}=0
$$

When the signal is stochastic and ms-differentiable, the flashing ratchet could be described by the following Langevin equation:

$$
\gamma \dot{x}=-\partial_{x}\left[V(x)\left(1+a_{\varsigma}(t)\right)\right]+\gamma \sqrt{2 D} \xi(t)
$$

where $a$ is a constant determining the depth of the modulation and $\varsigma(t)$ is the stochastic signal. To this the corresponding Fokker-Plank equation is:

$$
\begin{aligned}
& \partial_{t} P(x, t)+\partial_{x} j(x, t)=0, \\
& j(x, t)=-\frac{k T}{\gamma} \mathrm{e}^{-\frac{V(x)}{k T}} \partial_{x} \mathrm{e}^{\frac{V(x)}{k T}} P(x, t)-\frac{1}{2} \partial_{x}\left[s\left(\frac{F(x)}{\gamma}\right)^{2} P(x, t)\right]
\end{aligned}
$$

where

$$
s=\lim _{\Delta t \rightarrow 0} \frac{\left\langle\varsigma^{2}(t)\right\rangle_{t}}{\Delta t}
$$

In the case of ms-differentiable signal (23) is zero, [11].

$$
s=\lim _{\Delta t \rightarrow 0} \frac{\left\langle\varsigma^{2}(t)\right\rangle_{t}}{\Delta t}=0, \quad \text { for ms differenciable } \varsigma(t)
$$

In consequence of Equation (22) and the stationary process, the current density is zero again. Consequently, this machine is also non-workable.

When the signal is non-ms-derivable (like white noise, $\varsigma(t)$ ) than the last term of Equation (22) is non-zero, so in stationary conditions:

$$
\begin{aligned}
& \partial_{x} j(x, t)=0, \\
& j(x)=-\frac{k T}{\gamma} \mathrm{e}^{-\frac{V(x)}{k T}} \partial_{x} \mathrm{e}^{\frac{V(x)}{k T}} P(x)-\frac{1}{2} \partial_{x}\left[s\left(a \frac{F(x)}{\gamma}\right)^{2} P(x)\right] \\
& =\frac{F(x)}{\gamma} P(x)-\frac{k T}{\gamma} \partial_{x} P(x)-\frac{1}{2} \partial_{x}\left[s\left(a \frac{F(x)}{\gamma}\right)^{2} P(x)\right]
\end{aligned}
$$

In the last term the $F^{2}(x)$ jumps at the turning places of the potential, so the derivative (when $P(x)$ is there non-zero) is infinite, which has no physical meaning.

This means that the maximum of the bell-like $P(x)$ function must be con- 
siderably shifted from the turning point of the potential, assuring the finite solution. This makes possible the non-zero behavior of the current density. Indeed, the space average of Equation (25) is:

$$
\langle j(x)\rangle_{x}=\left\langle\frac{F(x)}{\gamma} P(x)\right\rangle_{x}-\frac{k T}{\gamma}\left\langle\partial_{x} P(x)\right\rangle_{x}-\frac{1}{2}\left\langle\partial_{x}\left[a s\left(\frac{F(x)}{\gamma}\right)^{2} P(x)\right]\right\rangle_{x}
$$

The last two terms of the right-hand side of the equation are zero, so

$$
\langle j(x)\rangle_{x}=\left\langle\frac{F(x)}{\gamma} P(x)\right\rangle_{x}
$$

\section{Discussion}

We had seen before that this term is zero when no modulation. It is however expected that with the same $F(x)$ and with shifted maximum place of $P(x)$ it will be non-zero. In the case of the potential of the saw-tooth function, the forces are constant in the linear intervals of the potential, so the stationary Fokker-Plank equation is transformed into an ordinary differential equation. When the force in the decreasing slope is $-F_{1}$, then for this interval from the second term of Equation (25) we obtain:

$$
\frac{F_{1}}{k T+\frac{1}{2 \gamma} s\left(a F_{1}\right)^{2}} \frac{\mathrm{d} P(x)}{\mathrm{d} x}+\frac{\mathrm{d}^{2} P(x)}{\mathrm{d} x^{2}}=0
$$

On the increasing slope, where the force is $F_{2}$, we have:

$$
\frac{F_{2}}{k T+\frac{1}{2 \gamma} s\left(a F_{2}\right)^{2}} \frac{\mathrm{d} P(x)}{\mathrm{d} x}-\frac{\mathrm{d}^{2} P(x)}{\mathrm{d} x^{2}}=0
$$

Equations (28) and (29) correspond to the results obtained elsewhere ([8] Equations (9a) and (9b)). From these above equations the consequences are identical:

- One of the most important is that the maximum position of $P(x)$ shifts right compared to the turning point of the potential, (Figure 3 ).

- The other consequence is that the dependence of the current density depends on the modulation depths that is resonant-like, [8].

The equations of the above discussed rigorous background of Brownian ratchets need numerical solutions.

\section{Conclusion}

The Feynman proposal for Brownian ratchet is incomplete; it could not demonstrate the Carnot-engine. In thermal equilibrium, time-average of the internal energy fluctuations is zero. The mistake was to ignore the fact that the Carnot cycle is a non-equilibrium process in a thermodynamic sense. While the Carnot engine works, the internal energy flows from the heat reservoir of higher temperature to lower one. In Feynman ratchet no such heat-flow is supposed. The 


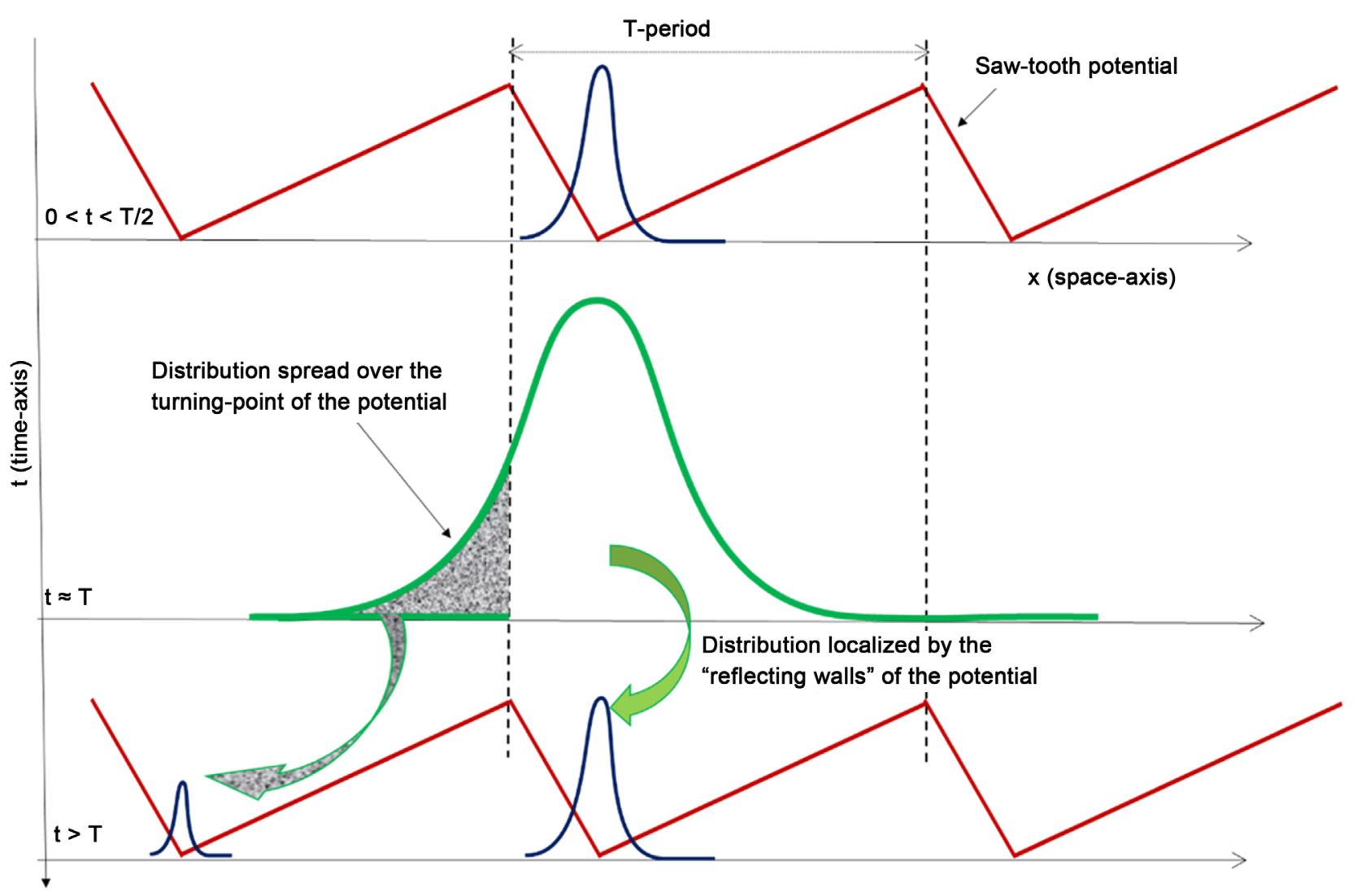

Figure 3. Explanation of the origin of the mass-current if the potential is on/off by T period of time. The particles at the beginning are situated in the vicinity of the minimum of the potential. When the potential is off, then the particles are symmetrically spread by diffusion. The subsequent switching on of the potential means that the remaining part of the particles (represented by the starting distribution) are located to the left-side minimum of the potential, while the majority part remains concentrated at the minimum-place. Consequently, a displacement and particle flow is oriented towards the right-hand side. The flow exists only when the distribution and the space-periodicity of the potential are comparable.

correct model is derived.

\section{Acknowledgements}

This work was supported by the Hungarian Competitiveness and Excellence Programme grant (NVKP_16-1-2016-0042).

\section{References}

[1] Astumian, R.D. and Derényi, I. (1998) Fluctuation Driven Transport and Models of Molecular Motors and Pumps. European Biophysics Journal, 27, 474-489. https://doi.org/10.1007/s002490050158

[2] Hänggi, P., Talkner, P. and Borkovec, M. (1990). Reaction-Rate Theory: Fifty Years after Kramers. Reviews of Modern Physics, 62, 251-341. https://doi.org/10.1103/RevModPhys.62.251

[3] Feynman, R.P., Leighton, R.B. and Sands, M. (1966) The Feynman Lectures on Physics. Adison-Wesley, California Institute of Technology, Reading.

[4] Tu, Z.C. (2008) Efficiency at Maximum Power of Feynman's Ratchet as a Heat Engine. The Journal of Physics A: Mathematical and Theoretical, 41, 312003. 
https://doi.org/10.1088/1751-8113/41/31/312003

[5] Van den Broeck, C., Meurs, P. and Kawai, R. (2005) From Maxwell Demon to Brownian Motor. New Journal of Physics, 7, 10-24.

https://doi.org/10.1088/1367-2630/7/1/010

[6] van der Meer, D., Eshuis, P., van der Weele, K. and Loshe, D. (2017) Realization of the Smoluchowski-Feynman Ratchet in a Granular Gas, XXII ICTAM, 25-29 August 2008, Adelaide.

http://extras.springer.com/2013/978-94-007-5967-1/CD/FSM05/11476.pdf.gz

[7] Eshuis, P., van der Weele, K., Loshe, D. and van der Meer, R.M. (2010) Experimental Realization of a Rotational Ratchet in a Granular Gas. Physical Review Letters, 104, 248001. https://doi.org/10.1103/PhysRevLett.104.248001

[8] Bier, M. and Astumian, D. (1996) Biased Brownian Motion as the Operating Principle for Microscopic Engines. Bioelectrochemistry and Bioenergetics, 39, 67-75. https://doi.org/10.1016/0302-4598(95)01833-6

[9] Curzon, F.L. and Ahlborn, B. (1975) Efficiency of Carnot Engine at Maximum Power Output. American Journal of Physics, 43, 22-24. https://doi.org/10.1119/1.10023

[10] Derényi, I. and Vicsek, T. (2001) Microscopic Mechanisms of Byological MotionFluctuations and Scaling in Biology. Oxford University Press, New York.

[11] Samuels, J.C. (1971) Elements of Stochastic Processes. In: Eringen, A.C., Ed., Continuum Physics, Academic Press, New York.

https://doi.org/10.1016/B978-0-12-240801-4.50011-X 